\title{
A primer on local food systems
}

\author{
Review by Amber A. Heckelman * \\ The University of British Columbia
}

Review of Selling L ocal: W hy L ocal F ood M ovements Matter, by Jennifer Meta Robinson and James Robert Farmer. (2017). Published by Indiana University Press. Available as paperback and ebook; 230 pages. Publisher's website: http:// www.iupress.indiana.edu/ product info.php? products id $=808693$

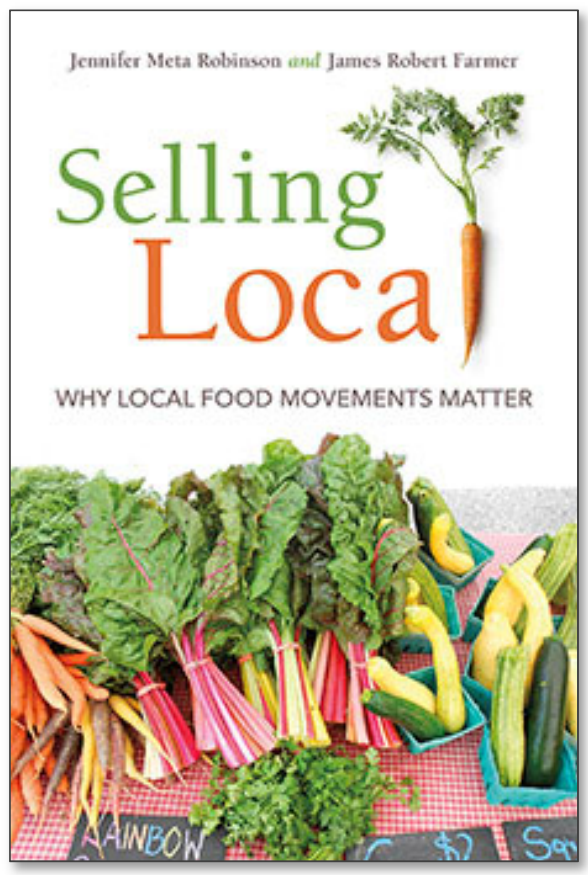

Submitted February 17, 2018 / Published online May 2, 2018

Citation: Heckelman, A. A. (2018). A primer on local food systems [Book review]. Journal of A griculture, F ood Systems, and C ommunity D evelopment, 8(2), 153-155. https:/ / doi.org/ 10.5304/ jafscd.2018.082.003

Copyright @ 2018 by the Author. Published by the Lyson Center for Civic Agriculture and Food Systems. Open access under CC BY license.

Tennifer Robinson and James Farmer's Selling L ocal: W hy L ocal F ood M ovements M atter consolidates decades of research on the local food movement, drawing attention to the array of local food developments in the U.S. Midwest and Appalachia regions. The authors provide a narrative that

\footnotetext{
* Amber A. Heckelman is a Bullitt Environmental Fellow, Liu Scholar, Public Scholar, and Ph.D. candidate with the Centre for Sustainable Food Systems at The University of British Columbia. Amber is committed to creating sustainable, equitable, and resilient food systems through knowledge translation and mobilization. Her research covers a range of topics that include climate resilience, food sovereignty, agroecology, and food assistance. She can be contacted at aheckelm@mail.ubc.ca.
}

weaves together voices from various stakeholders, taking the reader from farmers markets to community supported agriculture (CSA) to food hubs, while providing a scholarly analysis of the diverse capacities and limitations of these enterprises as well as offering a framework for assessing local food initiatives.

The title and content page hint at the underlying purpose of this book, which is to support the local food movement by identifying strengths, weaknesses, and leverage points that may be tapped to improve the capacity and success of diverse initiatives-all of which are necessary and important endeavors for cultivating and expanding local food systems. 
As a food system researcher, I was pleased that the authors contextualized the local food movement as a response to the increasingly globalized political economy, reflecting concerns with resource depletion and environmental degradation and also campaigns for social justice and equity. These issues are often addressed in vague or indiscernible terms in other works, but Robinson and Farmer use this context to provide both background and foreground to their narrative. This technique serves to maintain the reader's awareness of the complexity surrounding and occupying the local food space.

It was also refreshing to read a narrative that accounts for the role of historical processes and institutional mechanisms in creating the socioecological conditions that shape communities and characterize stakeholder experiences. The reader comes away with, for example, an understanding of how neoliberal economic policies sometimes compromise the ability of people, communities, and local producers and businesses to meet their needs by limiting access to educational opportunities and support services. Or how legacies of racism and discrimination remain prominent features in the food system landscape, in the form of barriers to land access and financial services and inequitable power dynamics within communities.

The authors provide many examples that illustrate why local food matters, describing how it (re)builds important connections that are favorable to communities, the environment, and our economies by fostering accountability, stewardship, and systems thinking. For instance, the many different iterations of local markets (e.g., crossroads, propriety, farmer-organized, public grower-vendor, and city renewal) are presented as spaces for citizenship, learning, creativity, community, and social responsibility. Similarly, the diverse iterations of CSAs and food hubs are also described as serving multiple purposes. And accompanying these descriptions are color photos depicting vibrant local food systems.

Readers interested in social-justice issues might be disappointed, however, as the discussion of social justice is mostly descriptive and less oriented toward suggesting pathways for addressing the historical configurations responsible for the inequalities that persist in these regions (e.g., differences in wealth, power, privilege, and resource access). The authors' acknowledgement of white privilege is noteworthy, but deserving of a more extensive consideration given the cries for equity and diversity in alternative agrifood movements- and in light of the current political climate, which is becoming increasingly hostile toward historically marginalized populations. There are some notable exceptions to this critique, however, as the authors do discuss some of the efforts being made to attract a broader demographic (beyond the white middle class currently occupying the local food space) through food literacy and diversity, food assistance programs, and alternative and progressive payment systems, to name a few. And there is an excellent critical discussion of how grassroots development initiatives do not necessarily result in improvements in equity and inclusion. D espite these contributions, readers are likely to be left grappling with how to effectively carryout social justice campaigns within their local food systems and the wider local food movement.

What is wonderful about this book is that Robinson and Farmer provide an intimate and comprehensive account of the local food movement that is unfolding in Bloomington, Indiana, and Huntington, West Virginia. The stories and reflections shared by community members and stakeholders bring the reader into the tensions, vibrancy, struggles, rewards, shortcomings, and triumphs that characterize the experiences of many local food proponents. Missing from the narrative, though, are voices of farmworkers and workers in the food-service industry; there is a very limited discussion of how their experiences and advocacies are being incorporated into the local food discourse in these regions.

In spite of this, the authors succeed in introducing readers to the nuances and intricacies of local food initiatives. Readers will come away with an understanding of the many dimensions (e.g., temporal, scale, social, environment, economic) of local and why the success of a local food system depends on the ability of proponents to account for its complexity and understand the socioecological conditions in which it is embedded. Readers will also come away feeling inspired and in awe of 
the many ways food shapes our lives, communities, and experiences in the world.

Robinson, a professor of practice in anthropology at Indiana University, and Farmer, an assistant professor in the School of Public Health at Indiana University, demonstrate their expertise in local food systems, providing readers with content that is both accessible and interesting to students, farmers, food system investors, and perhaps even conscious consumers. While this book likely will not satisfy veteran researchers looking for detailed statistics, it is well suited for novice researchers, educators, advocates, and anyone looking for a good primer on the evolving concept of local and its attributes in distinct sectors of the food system. Overall, the book is a valuable contribution to the local food movement. 